forme, la benzine et l'essence de pétrole; il est dédoublé par l'eau.

L'auteur croit en outre à la présence d'un ferment ne perdant pas son activité par l'alcool, la benzine et l'essence de pétrole mais la perdant par l'ether et le chloroforme.

\title{
Sur le dosage des alcaloïdes dans les écorces de quinquina.
}

PAR Mr. H. MEIJER ?).

L'auteur présente une critique de quelques méthodes, données par plusieurs chimistes pour doser l'ensemble des alcaloïdes contenus dans les écorces de quinquina et basées sur l'épuisement des écorces soit par les acides (acide chlorhydrique, acétique) soit par des corps alcalins (la potasse, la chaux).

En autre il indique une modification de la méthode de Mr. Moess, qui lui a donné de bons résultats.

Les méthodes que l'auteur a contrôlées expérimentalement sont celles de M.Mrs. Prollus ${ }^{2}$ ) 1re méthode), Moens ${ }^{3}$ ), DE $\mathrm{V}_{\mathrm{RIJ}}{ }^{4}$ ), ErkMan ${ }^{5}$ ), $\mathrm{H}_{\mathrm{AGER}}{ }^{6}$ ) et GunNing ${ }^{7}$ ).

Les conclusions, que l'auteur crôt pouvoir déduire de ses résultats, sont les suivantes:

$1^{0}$. En faisant bouillir l'écorce de quinquina en poudre

1) Archiv der Pharmacie. Bd. XVII. p. $721-736$ et $812-824$.

2) Archiv der Pharmacie. Bd. XVI. p. 85.

3) Haaxman's Tijdschrift voor de Pharmacie. 1875. p. 161.

4) Haaxman's Tijdschrift voor de Pharmacie. 1880, p. 18.

5) Hanuman's Tijdschrift voor de Pharmacie. 1881. p. 131.

6) Commentar zur Pharmacop. Germanic. 1er Theil. S. 527.

7) Maandblad voor Natuurwetenschappen. 1870. 
tenue avec de la chaux éteinte et de l'alcool à $90 \%$ pendant ' une heure, on extrait la quantité totale des alcaloïdes contenus dans l'écorce.

$2^{0}$. Une macération préalable de l'écorce avec l'acide sulfurique dilué ou avec de l'alcool aiguisé d'acide sulfurique, quelle qu'en soit la concentration, n'a aucune influence favorable sur le rendement en alcaloïde dans le cas où on emploie la chaux et l'alcool pour l'extraction des bases; elle n'a une valeur réelle que dans l'emploi des méthodes qui ne mènent pas à une extraction totale des alcaloïdes, (comme la méthode de Mr. Prollitus).

$3^{0}$. Dans le dosage des alcaloïdes il vaut mieux de séparer les alcaloïdes libres, on agitant le liquide alcalin avec de l'éther ou du chloroforme que de précipiter la base dans sa solution aqueuse par une matière alcaline (comme la soude.). $4^{0}$. La séparation de l'acide quinorique, de la quinorine et de la substance cireuse contenues dans le produit brut, obtenu par l'extraction avec l'alcool et la chaux, se fait sans perte en alcaloìde, si l'on ajoute à l'extrait alcoolique, avant l'évaporation, un excès d'acide sulfurique dilué, et en évaporant lentement, tandis qu'on agite le liquide. Les impuretés se présentent alors sous la forme de flocons légers, qui peuvent être lavés sans peine.

$5^{0}$. L'extraction totale des alcaloïdes n'est possible qu'en renouvelant à diverses reprises le traitement du mélange d'écorce et de chaux par l'alcool bouillant et qu'en prolongeant le système de dóplacement.

$6^{0}$. La méthode de Mr. Mozss, modifiée par l'auteur permet de donner dans l'espace de douze heures un résultat définitif quant à la teneur d'alcaloïde dans les écorces de quinquina.

$7^{0}$. Les méthodes d'extraction, qui se basent sur l'emploi des acides dilués (DE VRI, $\mathrm{H}_{\text {AGER}}$ ), d'un mélange de chloroforme et d'acide acétique absolu (ErkMav) ou d'un mélange de chloroforme, d'alcool et d'ammoniaque (Prollius) ne donnent pas de résultat satisfaisant; en employant ces méthodes 
on laisse toujours une certaine quantité d'alcaloïde dans l'ecorce pulvérisée.

$8^{0}$. Les méthodes de Mr. Gunnina et de Mr. Prollurus donnent des chiffres trop elevés pour la teneur en alcaloïde, parce que le produit final de l'analyse considéré comme un mélange pur de diverses bases, se compose en partie de quinovate $d \theta$ calcium et de matière cireuse.

Il est à remarquer que l'auteur n'a pas essayé la seconde méthode donnée par Mr. Pronitus (extraction de l'écorce par un mélange d'alcool, d'ether et d'ammoniaque), qui, dans la main de chimistes expérimentés a donné des résultats très-satisfaisants, surtout en $\mathrm{y}$ apportant la modification proposée par Mr. J. E. DE VRIJ (Voyez: Ce Journal. I. p. 68).

\section{Sur l'andromedotoxine, le princlpe vénéneux de l'Andromeda Japonien Thunb. par P. C. PLUGGE ').}

L'auteur donne une plns ample description de ses recherches sur l'andromedotoxine, commencées au Japon et poursuivies en Hollande, en employant des plantes, qu'il rapporta du Japon.

Après avoir observé les propriétés de l'andromedotoxine, qu'on trouve mentionnees dans l'extrait p. 224 de ce Recueil, il constata en outre que cette substance, contenue en plus grande quantité dans les feuilles de la plante que dans le bois, passe par le parchemin d'un dialyseur, puis qu'elle n'est pas emportóe par les vapeurs de l'eau bouillante, et enfin qu'elle n'est pas précipitée par les réactifs employés pour la recherche des alcaloïdes.

1) Tijdschrift voor Pharmacie in Nederland van HaAXMan en Legebeke, December 1882. 